\title{
DGP-News
}

\section{DGP-Kongress in Berlin}

Zum 56. Mal lud die Deutsche Gesellschaft für Pneumologie und Beatmungsmedizin (DGP) die lungenmedizinische Fachwelt zu ihrem großen Kongress. Die Tagung im Berliner CityCube stand unter dem Motto „Gemeinsam für den Patienten“. 591 Referentinnen und Referenten gaben ihr Wissen im Rahmen von fast 200 Veranstaltungen (Seminare, Kurse, Symposien und Vorträge) weiter. Die fast 4000 Besucher des Kongresses kamen während der vier Kongresstage zudem in den Genuss einer vielfältigen Ausstellung von Industrieunternehmen und weiteren Organisationen. Auf 4500 Quadratmetern Ausstellungsfläche stellten 135 Anbieter ihre Produkte und Dienstleistungen dem Publikum vor. Gut 170 Vertreter von Fach- und Publikumsmedien waren im CityCube vor Ort.

„Wir hatten einen sehr erfolgreichen Kongress, der sehr gut von den Teilnehmern angenommen wurde“, bilanzierte der neue DGP-Präsident Berthold Jany, Professor und Chefarzt an der Missionsärztlichen Klinik in Würzburg. „Das Motto von Tagungspräsident Torsten Bauer ist sehr gut in vielen Symposien und Veranstaltungen vermittelt worden. Der Patient stand im Mittelpunkt.“ Zudem ging es hier um vernetzte Strukturen, sektorübergreifende Behandlung und die Schnittstelle

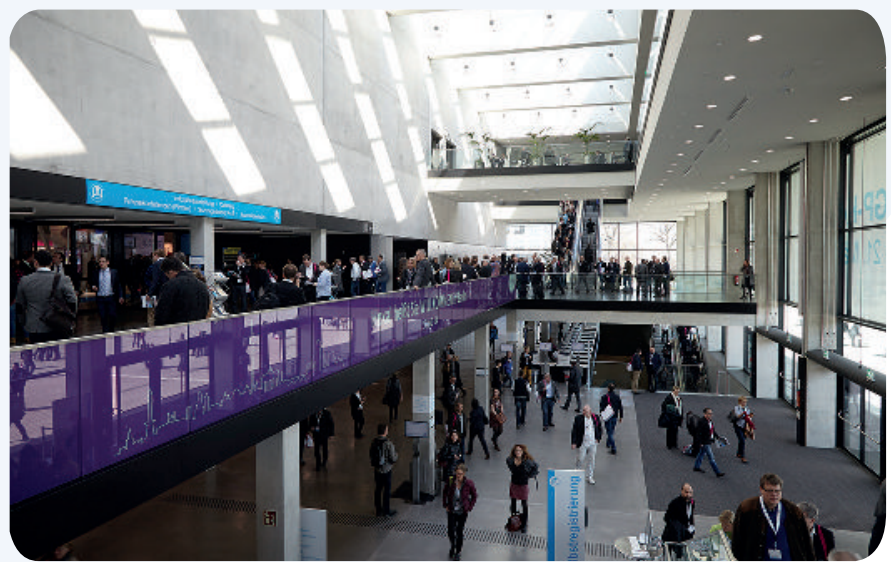

Gemeinsam für den Patienten: der DGP-Kongress 2015 im CityCube Berlin zwischen ambulant und stationär. In drei zentralen Pressekonferenzen wurden weitere spezielle pneumologische Themen beleuchtet.

In einer großen Eröffnungs-Pressekonferenz begrüßten am ersten Kongresstag der dann noch amtierende DGP-Präsident Tobias Welte, sowie Kongresspräsident Torsten Bauer und Gunda Leschber, Vizepräsidentin der Deutschen Gesellschaft für Thoraxchirurgie, die Pressevertreter im Berliner CityCube. Das moderne, transparente Ambiente von DGP-Stand- und Pressefläche sei dabei sinnbildlich für das aktuelle Wirken der Pneumologen, erklärte Professor Welte. In drei Themenblöcken wurde $\mathrm{zu}$ den Themen „E-Zigarette“, „Prävalenz von Lungenkrankheiten“ und „Lungensport“ berichtet. Besondere Höhepunkte waren die Vorstellung des Positionspapiers zur elektronischen Zigarette und sowie die Praxisberichte von Leichtathletin Franziska Liebhardt (33), die sich mit transplantierter Lunge auf die Olympischen Spiele 2016 in Rio de Janeiro vorbereitet. Das Positionspapier zur E-Zigarette hatte die DGP zuvor in Zusammenarbeit mit neun anderen Fachgesellschaften und medizinischen Organisationen erstellt und sorgte bereits unmittelbar nach Veröffentlichung in der Öffentlichkeit für Diskussionsstoff.

Auch am zweiten Kongresstag drehte sich vieles um die E-Zigarette. Professor Claus Vogelmeier, neuer Vorsitzender der Deutschen Lungenstiftung (DLS), stellte auf einer weiteren Pressekonferenz der DGP dar, wie sich der Konsum von E-Zigaret- ten verändert. Eine Regulierung durch den Gesetzgeber werde wohl noch eine lange Zeit beanspruchen, glaubt Vogelmeier. Dies habe schließlich auch der Kampf gegen den Zigarettengebrauch gezeigt.

In einer abschließenden DGP-Pressekonferenz, gemeinsam mit dem Zentralkomitee zur Bekämpfung der Tuberkulose (DZK), ging es neben den aktuellsten Entwicklungen zum Thema „Lungenkrebs“ auch um Tuberkulose am speziellen Beispiel von Migranten. Auch wenn der Anteil Erkrankter mit Migrationshintergrund zunehmend steige, erklärte Martin Priwitzer vom Stuttgarter Gesundheitsamt dazu, könne man nicht automatisch daraus schließen, Flüchtlinge schleppten die Tuberkulose nach Deutschland. Karl Schenkel, Mitarbeiter des DZK, lieferte weitere Details zur weltweiten Tuberkuloselage. In einer interessanten Fragerunde ging es außerdem vertiefend um die besondere Gruppe inhaftierter Straftäter und den Umgang mit ihnen im Zusammenhang mit Tuberkulose.

Auf dem Kongress in Berlin hat die DGP gemeinsam mit DLS und DZK zudem erstmals in einer gemeinsamen Veranstaltung zahlreiche Ehrungen in verschiedenen Kategorien vorgenommen. Zwölf Preisträger wurden für ihre wissenschaftlichen und journalistischen Arbeiten im Rahmen der mit insgesamt fast 40000 Euro dotierten Feier „Best of Pneumology“ ausgezeichnet.

In Leipzig wird man sich 2016 wieder treffen. DGP-Präsident Berthold Jany erwartet dort erneut ein „stimulierendes Meeting“. Dort hat Tagungspräsident Hubert Wirtz den Leitgedanken „Innovative Pneumologie“ formuliert. Das Motto „Gemeinsam für den Patienten“ wird allerdings auch in Leipzig ein zentrales Thema bleiben. Jany: „Das Motto bleibt - und zwar für immer.“ 